\title{
Quantifying non-Markovianity in underdamped versus overdamped environments and its effect on spectral lineshape.
}

\author{
Dale Green, ${ }^{1}$ Ben S. Humphries, ${ }^{1}$ Arend G. Dijkstra, ${ }^{2}$ and Garth A. Jones ${ }^{1}$, a) \\ ${ }^{1)}$ School of Chemistry, University of East Anglia, Norwich Research Park, Norwich, NR4 7TJ, \\ $U K$ \\ 2) School of Chemistry and School of Physics and Astronomy, University of Leeds, Leeds, LS2 9JT, \\ $U K$
}

(Dated: 18 October 2019)

Non-Markovian effects in open quantum systems are central to understanding spectral lineshape. Here, we quantify the non-Markovianity associated with both overdamped and underdamped vibrations in terms of information flow between the bath and the system and compare this with the broadening and ellipticity of two-dimensional spectra. Using the Breuer Laine Piilo (BLP) measure, we link well-known stochastic models for spectral lineshape with modern quantum information theory. Specifically we study the effect of nonMarkovianity in a system in contact with underdamped vibrations and examine the differences observed on increasing the damping to the overdamped limit. The open quantum system dynamics are evolved using the hierarchical equations of motion, efficiently terminated with a Markovian cut-off, where separate hierarchies are derived for the underdamped and overdamped environments. It is shown that the BLP measure is quantitatively correlated with the ellipticity of two-dimensional spectra and memory effects are more pronounced in underdamped environments, due to the long-lived feedback of information between the system and its bath, compared to overdamped environments. Environmental signatures in spectral lineshapes emerge as a result of information flow from the bath back into the system.

Keywords: non-Markovian, HEOM, trace distance, BLP measure, 2D Spectroscopy, underdamped, energy transfer

\section{INTRODUCTION}

Condensed phase spectroscopy, in its various forms, has found wide application in chemistry and physics not only for its usefulness in probing the chemical structure of solute molecules, but for also providing detailed information of the solvent dynamics via the spectral lineshape 15 In linear spectroscopy, inhomogeneous and homogeneous broadening are mixed on a single spectral axis! ${ }^{6}$ Two-dimensional (2D) spectroscopy overcomes this by separating the inhomogeneous and homogeneous contributions with the use of a four-wave mixing procedure; the inhomogeneous component being projected onto the diagonal axis and the homogeneous onto the anti-diagonal. ${ }^{3 / 7 / 8}$ The two-dimensional lineshape depends sensitively on the time scale of interactions of the chromophore probed with the environment. Homogenenous and inhomogeneous broadening are the short and long time limits, respectively, but, if the system-bath interaction time scale is not in either limit, the spectral lineshape will be sensitive to the time scale. In this case, it is necessary to describe the history of the bath. In order to microscopically model $2 \mathrm{D}$ spectroscopy experiments with accurately broadened peaks, one must have confidence that the theoretical framework being employed can describe this history sufficiently well, and in-turn, that the state of the bath over this history influences the dynamics of the quantum system correctly.

\footnotetext{
a) Electronic mail: garth.jones@uea.ac.uk
}

Accurately modelling the interactions of a bath with a quantum system of interest presents a significant challenge and over the years, highly sophisticated open quantum system methodologies have been developed. ${ }^{9] 10}$ Generally, the quantum system of interest is treated explicitly and is distinguished from the bath degrees of freedom, which are modelled stochastically, often as an ensemble of harmonic oscillators defined via a spectral density! 1112 There are a number of perturbative approaches for modelling these types of systems that allow one to calculate adequate spectral lineshapes phenomenologically! 113 However, it is often desirable to use a more physically realistic microscopic model, whereby one can evolve the reduced density matrix of the system subject to a given Hamiltonian within a non-perturbative formalism. One of the most commonly employed non-perturbative approaches in recent years is the hierarchical equations of motion (HEOM), originally developed by Tanimura and co-workers! 14 16 Here the propagation of an hierarchy of auxiliary density operators (ADOs) accounts for the quantum system's memory of the bath. HEOM methods for a variety of spectral densities have found numerous applications, including studies of electron transfer and exciton dynamics in molecular aggregates. $17+\sqrt{20}$

Dynamically, the memory of the bath can be understood in terms of the rate of relaxation of the system relative to that of its surrounding environment. The significance of the bath memory effects to the system dynamics is often referred to as the degree of non-Markovianity, in analogy to classical probability theory. ${ }^{21}$ In the Markovian limit, the fluctuations of the bath are much faster than those of the system and consequently the open 
quantum system evolves with no memory of the state of the bath at earlier times ${ }^{[0 \mid 22]}$ On the other hand, in non-Markovian cases, the quantum system and the bath oscillate on similar time scales and the system dynamics are dependent upon some memory of the state of the bath at earlier times. ${ }^{23}$ Therefore, it is essential to incorporate the influence of the bath on the system over some finite history, when evolving the reduced density matrix of the system.

More rigorously, one can understand these limits in terms of the flow of information between the system and bath; information within this context corresponding to the distinguishability of one quantum system from another ${ }^{24 \mid 25}$ In the Markovian limit, information flows unidirectionally from the system to the bath. The faster bath oscillations mean that information is irreversibly lost from the system to the bath. In the nonMarkovian case, information can be transferred from the bath back into the system. ${ }^{26}$ The similar system and bath time scales providing opportunity for information recently transferred to the bath to return to the system before it is dissipated further. Note that because nonMarkovian evolution allows information to flow in both directions, it implicitly includes Markovian evolution as a limiting case.

For our purposes, the open quantum system corresponds to the vibrational motion of a thermodynamic ensemble, where the solute chromophore is the quantum system of interest and the solvent, its bath. Coupling to the bath degrees of freedom causes the vibrational motion of the system to become damped, leading to dissipation and dephasing $\left[\frac{15}{15}\right.$ This has consequences for the wider system dynamics, particularly after undergoing perturbation by external forces, such as the electric field of a laser pulse. It is therefore the bath damping which ultimately determines the lineshape in spectroscopy. Minimal damping allows the full vibrational structure to be detected, whilst significant damping leads to the homogeneous and inhomogeneous limits. ${ }^{[27}$ The homogeneous limit, associated with strong damping but where the bath relaxation is still fast compared with that of the system, results in identical system-bath interactions across the ensemble $\frac{15}{15}$ That is to say, the individual solute molecules become entirely indistinguishable. Whereas in the inhomogeneous, or static, limit the solute molecules exist in a broader distribution of states, due to much slower bath motion caused by the strongest damping [3]

The objective of this work is to understand experimental spectral lineshape in terms of information flow and memory effects, from a microscopic perspective, between a quantum system and its bath. We measure the degree of non-Markovianity for a simple two level system whose vibration is damped by a harmonic bath, in both the underdamped and overdamped limits. The affect of increased damping on the measurable nonMarkovianity is assessed by modelling the return to equilibrium of the system after perturbation by an external laser field. Comparison with calculated linear absorp- tion and 2D photon echo spectra for each of the damping limits demonstrates the importance of non-Markovian effects in determining spectral lineshape. This is achieved through application of separate HEOM methods derived specifically for the underdamped and overdamped limits, both terminated with an efficient Markovian convergence parameter. Our results show that theoretically calculated non-Markovianity defined by the BLP measure is directly correlated with the ellipticity in the twodimensional spectra, which is experimentally observable.

\section{MEASURING NON-MARKOVIANITY}

Interactions between the chromophore and its environment correspond to the exchange of information between the system and bath along a quantum channel. ${ }^{21} \mathrm{In}$ the language of quantum information theory, the quantum channel is an operator through which information is transferred. As a consequence, a quantum channel represents an exact description of the evolution of the density operator.

The Von-Neumann entropy, a functional capable of describing a mixed state, links bulk thermodynamic properties with discrete bits of quantum information. ${ }^{[28}$ When there is a maximal knowledge of the system it is in a pure quantum state with zero entropy. Conversely, if there is less than complete knowledge of the system, then it is a mixed state, where the magnitude of information is proportional to the number of microstates and inversely proportional to their respective weights. ${ }^{[29}$ Given a density matrix, $\rho$, for an ensemble of system particles, we can write the entropy functional as $S(\rho) \equiv-k_{\mathrm{B}} \operatorname{Tr}(\rho \ln \rho) \frac{9}{9}$ Crucially, the entropy functional is zero if and only if $\rho$ is a pure state.

For a Markovian process, the future state of the density matrix is dependent only on the present state. It is intuitive for a process that follows the Markov property to have a concave entropy functional, $\rho \mapsto S(\rho)$, such that $S\left(\sum_{i} \lambda_{i} \rho_{i}\right) \geq \sum_{i} \lambda_{i} S\left(\rho_{i}\right)$, for non-vanishing $\lambda_{i} \cdot{ }^{9128}$ Furthermore, when the system is composite, there is a subadditivity condition: $\mathcal{H}=\mathcal{H}^{(1)} \otimes \mathcal{H}^{(2)}$ where $S(\rho) \leq$ $S\left(\rho^{(1)}\right)+S\left(\rho^{(2)}\right)$. Consequently, during a Markovian evolution, the system is monotonically losing information to the environment, as each successive state evolves independently of its history towards equilibrium. $10121 / 22$ Monotonic loss of information for quantum states is defined by decreasing distinguishability of different quantum systems with time.24

The intrinsic relationship between the maximal values of information flux, entropy and distinguishability of particles is manifest in the trace distance. For a pair of states, $\rho_{1}$ and $\rho_{2}$, the trace distance is defined as, $22|26| 30$

$$
D\left(\rho_{1}, \rho_{2}\right)=\frac{1}{2} \operatorname{Tr}\left|\rho_{1}-\rho_{2}\right|,
$$

where $|A|=\left(A^{\dagger} A\right)^{1 / 2}$. The metric is constructed in this way to ensure that the supports of the kernel matrix are 
orthogonal when the flux of information is negative, corresponding to an increase in entropy of the system and a loss of distinguishability. The factor of half denotes that each state is equally probable, and the matrix half power is defined by diagonalization. The maximum and minimum values of the trace distance are observed when the supports are orthogonal or parallel, respectively. Physically this corresponds to completely distinguishable and completely indistinguishable quantum states.

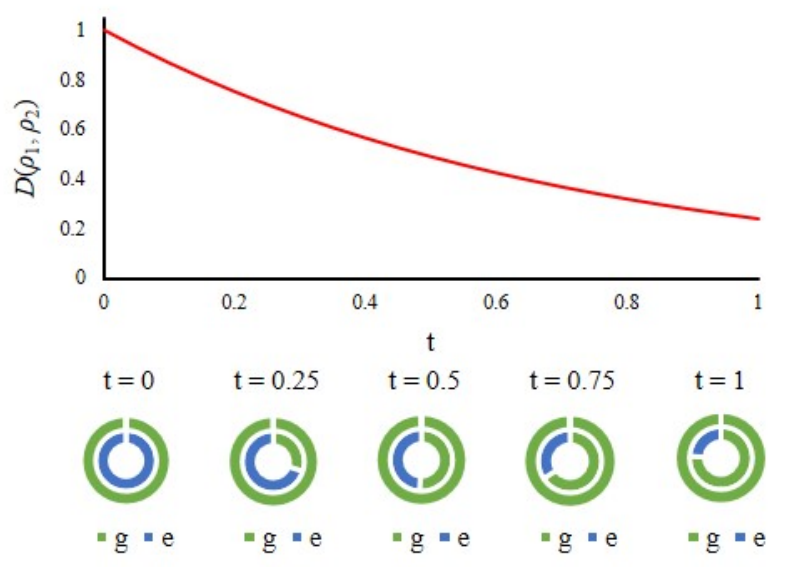

FIG. 1. The trace distance, $D\left(\rho_{1}, \rho_{2}\right)$, for a two level system initially in its ground state (outer) compared with its excited state (inner) decreases as the excited state population relaxes, following the decrease in distinguishability.

A demonstration of the trace distance is given in figure 1 for a simple two level system with orthogonal initial conditions. The nested pie charts show the populations of the ground (g) and excited (e) states at specific times, where the outer (inner) chart corresponds to an initial population entirely in its ground (excited) state. As time progresses, the excited state irreversibly dissipates energy into the bath, increasing the ground state population, leading to a decrease in distinguishability between the two systems, resulting in a decrease in the trace distance.

Using this definition of distinguishability, it is possible to construct a measure of non-Markovianity.24 Changes in the distinguishability of quantum systems are due to changes in the values of bits of information as a function of time and hence the key dynamical property is the flux of information between the system of interest and the bath, $\sigma ! 31$

$$
\sigma=\frac{\mathrm{d}}{\mathrm{d} t} D\left(\rho_{1}, \rho_{2}\right)=\frac{\mathrm{d}}{\mathrm{d} t}\left(\frac{1}{2} \operatorname{Tr}\left|\rho_{1}-\rho_{2}\right|\right) .
$$

Markovianity requires a divisible dynamical map from the time convolutionless master equation, $\Lambda(t+\tau, 0)=$ $\Lambda(t+\tau, t) \Lambda(t, 0)$, and a monotonic loss of information to the environment. 9 This means that a non-Markovian process must correspond to a strictly positive flux, $\sigma>0$. However, divisibility is not a necessary condition for negative flux. That is to say, a negative flux is a neces- sary but not sufficient condition for Markovianity. Consequently, we take the information flux for our system and set every negative value to zero leaving the purely non-Markovian contributions. Then by integrating over time for the maximum positive flux in the system, we can define a magnitude for the information returned to the system and the degree of non-Markovianity. The BLP measure, $\mathcal{N}$, has been used previously to quantify the non-Markovianity of a general quantum system in this way, $24|26| 30|32| 33$

$$
\mathcal{N}=\max _{\rho_{s}^{1,2}} \int_{\sigma>0} \sigma(t) \mathrm{d} t
$$

Therefore, a non-Markovian evolution has a strictly non-zero information flux and a quantifiable nonMarkovianity of $\mathcal{N}$. Through this measure, we can relate microscopic non-Markovianity with macroscopic spectral properties.

\section{THEORETICAL MODEL}

We define a two-level-system with a ground, $|g\rangle$, and excited, $|e\rangle$, state as

$$
\hat{H}_{S}=-\frac{\hbar \omega_{e g}}{2} \sigma_{z}
$$

where $\tilde{\omega}_{e g}=3000 \mathrm{~cm}^{-1}$ and the dipole moment operator $\hat{\mu}=\sigma_{x}$. The electronic states of the system are coupled to a single vibrational mode of frequency $\tilde{\omega}_{0}=500 \mathrm{~cm}^{-1}$, whose coordinates are in turn coupled to those of the environment, which is modelled as a bath of harmonic oscillators representing phonon modes $\frac{34}{4}$ Assuming a continuum of bath modes with masses, $m_{\alpha}$, and frequencies, $\omega_{\alpha}$, the distribution of coupling strengths, $g_{\alpha}$ is determined by the spectral density, $J(\omega) \underline{35136}$

$$
J(\omega)=\sum_{\alpha} \frac{g_{\alpha}^{2}}{2 m_{\alpha} \omega_{\alpha}} \delta\left(\omega-\omega_{\alpha}\right) .
$$

Through a canonical transformation, the vibrational mode of the system is subsumed into the bath degrees of freedom, creating a Brownian oscillator which couples directly to the electronic states of the system and is damped by the motion of the wider environment through the parameter $\gamma \underline{34}$ The system couples to the bath through the operator,

$$
\hat{V}_{S}=\sigma_{x}-\frac{\sigma_{z}}{2}
$$

accounting for both dephasing, $\sigma_{z}$, and dissipation, $\sigma_{x}$ processes. $23|37| 38$ Here, dephasing refers to the decoherence of wavepackets due to the stochastic perturbation of the system potential energy surface by the bath modes, causing the system transition frequency to deviate from its equilibrium value. Dissipation refers to the relaxation 
of the system as excitation energy is transferred to the bath degrees of freedom.

When $2 \omega_{0} \gg \gamma$, the Brownian oscillator is underdamped and has the spectral density,

$$
J(\omega)=\frac{2 \hbar \eta \gamma \omega_{0}^{2} \omega}{\left(\omega_{0}^{2}-\omega^{2}\right)^{2}+\gamma^{2} \omega^{2}},
$$

which features a peak at the mode frequency, with a width controlled by $\gamma^{[39}$ The strength of the system-bath coupling is determined by the reorganisation energy, $\eta$, which is temperature dependent,

$$
\eta=\frac{\hbar \Delta^{2}}{2 k_{\mathrm{B}} T}
$$

In terms of dephasing, $\Delta$ is the fluctuation amplitude, a measure of the range over which the transition frequency of the system fluctuates. Stronger system-bath coupling leads to greater deviation from the equilibrium transition frequency and faster dephasing.

The influence of the entire bath degrees of freedom is captured within the system-bath correlation function,

$$
C(t)=\frac{1}{\pi} \int_{0}^{\infty} \mathrm{d} \omega J(\omega)\left(\operatorname{coth}\left(\frac{\beta \hbar \omega}{2}\right) \cos \omega t-i \sin \omega t\right),
$$

where $\beta=\left(k_{\mathrm{B}} T\right)^{-1} \underline{14139}$ This obeys the fluctuationdissipation theorem, where the imaginary component causes dissipation and the real component is responsible for thermally induced fluctuations. $\frac{36 / 40}{36}$

The solution to the correlation function is the sum of exponential terms, containing the bosonic Matsubara frequencies, $\nu_{k} ; k=0,1,2, \ldots, M$, and prefactors, $c_{k}, \frac{1139}{,}$

$$
C(t)=\sum_{k=0}^{M} c_{k} e^{-\nu_{k} t}
$$

where, with $\zeta=\sqrt{\omega_{0}^{2}-\frac{\gamma^{2}}{4}}$,

$$
\begin{aligned}
& \nu_{0}=\frac{\gamma}{2}-i \zeta, \\
& \nu_{1}=\frac{\gamma}{2}+i \zeta, \\
& \nu_{k}=\frac{2 \pi(k-1)}{\hbar \beta}, \\
& c_{0}=\frac{\hbar \eta \omega_{0}^{2}}{2 \zeta}\left\{\operatorname{coth}\left(\frac{\hbar \beta}{2}\left(\zeta+i \frac{\gamma}{2}\right)\right)-1\right\}, \\
& c_{1}=\frac{\hbar \eta \omega_{0}^{2}}{2 \zeta}\left\{1-\operatorname{coth}\left(\frac{\hbar \beta}{2}\left(-\zeta+i \frac{\gamma}{2}\right)\right)\right\}, \\
& c_{k}=-\frac{4 \eta \gamma \omega_{0}^{2}}{\hbar \beta} \frac{\nu_{k}}{\left(\omega_{0}^{2}+\nu_{k}^{2}\right)^{2}-\gamma^{2} \nu_{k}^{2}} .
\end{aligned}
$$

An hierarchy of auxiliary density operators (ADOs) can then be derived and propagated using,

$$
\begin{aligned}
\dot{\rho}_{\mathbf{j}}(t)= & -\left(\frac{i}{\hbar} \hat{H}_{S}^{\times}+\sum_{k=0}^{M} j_{k} \nu_{k}-\sum_{k=M}^{\infty} \hat{V}_{S}^{\times} \hat{\Psi}_{k}\right) \rho_{\mathbf{j}}(t) \\
& +\sum_{k=0}^{M} \hat{V}_{S}^{\times} \rho_{j_{k}^{+}}(t)+j_{0} \hat{\Theta}^{-} \rho_{j_{0}^{-}}(t)+j_{1} \hat{\Theta}^{+} \rho_{j_{1}^{-}}(t) \\
& +\sum_{k=2}^{M} j_{k} \nu_{k} \hat{\Psi}_{k} \rho_{j_{k}^{-}}(t) \\
= & \mathcal{L} \rho_{\mathbf{j}}(t),
\end{aligned}
$$

where

$$
\begin{aligned}
\hat{\Psi}_{k} & =\frac{4 \eta}{\hbar \beta} \frac{\gamma \omega_{0}^{2}}{\left(\omega_{0}^{2}+\nu_{k}^{2}\right)^{2}-\gamma^{2} \nu_{k}^{2}} \hat{V}_{S}^{\times} \\
\hat{\Theta}^{ \pm} & =\frac{\eta \omega_{0}^{2}}{2 \zeta}\left\{\mp \hat{V}_{S}^{\circ} \pm \operatorname{coth}\left(\frac{\hbar \beta}{2}\left(\mp \zeta+i \frac{\gamma}{2}\right)\right) \hat{V}_{S}^{\times}\right\}
\end{aligned}
$$

and $\hat{V}_{S}^{\times} \rho=\left[\hat{V}_{S}, \rho\right]$ denotes the commutator of the bath coupling operator and the density matrix and $\hat{V}_{S}^{\circ} \rho=$ $\left\{\hat{V}_{S}, \rho\right\}$, the anti-commutator ${ }^{35}$

The ADOs are identified by the $(M+1)$-dimensional vectors $\mathbf{j}=\left(j_{0}, \ldots, j_{k}, \ldots, j_{M}\right)$ where the elements, $j_{k}$, are integer multipliers of each Matsubara frequency. The true reduced density matrix of the system corresponds to $\rho_{\mathbf{0}}$, with all coefficients equal to zero. The hierarchical propagator connects the ADOs through the terms involving $\mathbf{j}^{ \pm}=\left(j_{0}, \ldots, j_{k} \pm 1, \ldots, j_{M}\right)$, accounting for system-bath correlations which enable memory effects to be incorporated into the dynamical evolution. 37

The hierarchy is terminated using a convergence parameter, $\xi$, beyond which the evolution is assumed to be within the Markovian limit 36141 The convergence parameter determines the number of Matsubara frequencies via,

$$
\frac{2(M+1) \pi}{\hbar \beta}>\xi
$$

and the hierarchy depth according to,

$$
\sum_{k=0}^{M} j_{k}\left|\operatorname{Re}\left(\nu_{k}\right)\right|>\xi
$$

As discussed by Dijkstra and Prokhorenko, this truncation scheme is more efficient than previous methods as long as $\xi$ is larger than the energy scales of the system, because ADOs in directions with larger $\nu$ are terminated more quickly, which reduces the total number of ADOs while retaining accuracy ${ }^{36}$ Terminating ADOs are propagated using the approximation,

$$
\dot{\rho}_{\mathbf{j}}(t) \simeq-\left(\frac{i}{\hbar} \hat{H}_{S}^{\times}+i\left(j_{0}-j_{1}\right) \zeta-\sum_{k=M}^{\infty} \hat{V}_{S}^{\times} \hat{\Psi}_{k}\right) \rho_{\mathbf{j}}(t),
$$

where the sum to infinity is truncated at a suitably large value ${ }^{35}$ 
The above HEOM can also be used for the overdamped case, when $2 \omega_{0} \ll \gamma$ and $\zeta=i \sqrt{\frac{\gamma^{2}}{4}-\omega_{0}^{2}}$, but can be simplified via a change of the spectral density 1 An overdamped Brownian oscillator has the Debye spectral density,

$$
J(\omega)=2 \eta \frac{\omega \Lambda}{\omega^{2}+\Lambda^{2}},
$$

which concentrates the greatest coupling strength at lower frequency phonon modes, dominated by a peak at,

$$
\Lambda=\frac{\omega_{0}^{2}}{\gamma}=\frac{1}{\tau_{c}},
$$

which defines the correlation time, $\tau_{c}$, of the bath correlation function, equal to the time required for the bath to return to equilibrium upon perturbation! 110

In order to investigate non-Markovianity, we assume that the time scale for the system to return to equilibrium, $T_{S}$ is longer than that of the bath, but both are shorter than the natural recurrence of the bath oscillations, $\tau_{R}$, such that $\tau_{c} \ll T_{S} \ll \tau_{R}$. This is true for continuous spectral densities, where the great range of frequencies increases $\tau_{R} \frac{10}{10}$ When the bath correlation time is much shorter than the system time scale, $\tau_{c} \ll T_{S}$, information transferred to the bath degrees of freedom is quickly dissipated and the evolution approximately Markovian. But as the correlation time increases, approaching the system time scale, $\tau_{c} \approx T_{S}$, there is greater opportunity for non-Markovian information transfer back and forth between the system and bath.

In the overdamped case, the solution to the correlation function remains the sum of exponential terms, as in eq. 10, where,

$$
\begin{aligned}
\nu_{0} & =\Lambda, \\
\nu_{k} & =\frac{2 \pi k}{\hbar \beta}, \\
c_{0} & =\eta \Lambda\left(\cot \left(\frac{\hbar \beta \Lambda}{2}\right)-i\right), \\
c_{k} & =\frac{4 \eta \Lambda}{\hbar \beta}\left(\frac{\nu_{k}}{\nu_{k}^{2}-\Lambda^{2}}\right),
\end{aligned}
$$

and the hierarchical propagator has the form,, $36 \mid 41$

$$
\begin{aligned}
\dot{\rho}_{\mathbf{j}}(t)= & -\left(\frac{i}{\hbar} \hat{H}_{S}^{\times}+\sum_{k=0}^{M} j_{k} \nu_{k}\right) \rho_{\mathbf{j}}(t)-i \sum_{k=0}^{M} \hat{V}_{S}^{\times} \rho_{j_{k}^{+}}(t) \\
& -i \sum_{k=0}^{M} j_{k}\left(c_{k} \hat{V}_{S} \rho_{j_{k}^{-}}(t)-c_{k}^{*} \rho_{j_{k}^{-}}(t) \hat{V}_{S}\right) \\
& -\left(\frac{2 \eta}{\hbar \beta \Lambda}-\eta \cot \left(\frac{\hbar \beta \Lambda}{2}\right)-\sum_{k=1}^{M} \frac{c_{k}}{\nu_{k}}\right) \hat{V}_{S}^{\times} \hat{V}_{S}^{\times} \rho_{\mathbf{j}}(t) \\
= & \mathcal{L} \rho_{\mathbf{j}}(t) .
\end{aligned}
$$

This hierarchy is terminated as before, where terminating ADOs are propagated using the standard von Neumann form,

$$
\dot{\rho}_{\mathbf{j}}(t) \simeq-\frac{i}{\hbar} \hat{H}_{S}^{\times} \rho_{\mathbf{j}}(t) .
$$

The bath correlation time, $\tau_{c}$, creates two additional limits within the overdamped case, determined by the dimensionless parameter $\Delta \cdot \tau_{c}$, valid in the high temperature limit of $k_{\mathrm{B}} T \gg \hbar \Lambda ! 1$ Short correlation times compared with the fluctuation amplitude produce the homogeneous limit, $\Delta \cdot \tau_{c} \ll 1$, where the system-bath interaction is identical across the entire ensemble ${ }^{38}$ Whereas long correlation times produce the inhomogeneous limit, $\Delta \cdot \tau_{c} \gg 1$, where a slower return to equilibrium enables the detection of differences within the system-bath interaction across the ensemble.$^{7}$ These have noticeable effects on spectral lineshape, as discussed in section IV]

Here we set $\tilde{\eta}=20 \mathrm{~cm}^{-1}$, such that $\tilde{\Delta}=91.33 \mathrm{~cm}^{-1}$, at $300 \mathrm{~K}$. Note that we will consistently use the tilde to denote conversion of a parameter to wavenumbers such that $\tilde{\eta}=\eta(2 \pi c)^{-1}$ etc. This small reorganisation energy corresponds to a weak displacement, with a Huang-Rhys factor of $S=\eta / \omega_{0}=0.04$. The range of damping strengths used in these simulations are presented in table I] along with their respective dissipation/dephasing rates, $\Lambda$, correlation times, $\tau_{c}$, and $\Delta \cdot \tau_{c}$ values for the overdamped cases. Increasing the damping strength decreases the dissipation rate, slowing the system-bath interactions and increasing the correlation time, and shows a progression from the underdamped, to the homogeneous overdamped and finally to the inhomogeneous overdamped limits.

\begin{tabular}{c|c|c|c}
\hline$\tilde{\gamma} / \mathrm{cm}^{-1}$ & $\Lambda(2 \pi)^{-1} / \mathrm{fs}^{-1}$ & $\tau_{c} / \mathrm{fs}$ & $\Delta \cdot \tau_{c}$ \\
\hline 50 & 0.150 & 7 & - \\
300 & 0.025 & 40 & - \\
1750 & 0.004 & 234 & 0.64 \\
2750 & 0.003 & 367 & 1.00 \\
8200 & 0.001 & 1094 & 3.00 \\
\hline
\end{tabular}

TABLE I. Damping strengths, dissipation rates and correlation times used, for $\tilde{\eta}=20 \mathrm{~cm}^{-1}$ such that $\tilde{\Delta}=91.33 \mathrm{~cm}^{-1}$ at $300 \mathrm{~K} . \Delta \cdot \tau_{c}$ given for overdamped environments only.

To calculate $\mathcal{N}$ for each damping strength, we compare the dynamics of the system with and without excitation due to a Gaussian laser pulse. Adopting the semiclassical approximation for a real laser field, the systemfield interaction Hamiltonian is given by, $42 \mid 43$

$$
\begin{aligned}
\hat{H}_{S F}(t) & =-\hat{\mu} \cdot \mathcal{E}(\mathbf{r}, t) \\
& =-\hat{\mu} \cdot\left(\chi E\left(t-\tau_{p}\right) \exp (-i \omega t+i \mathbf{k r})\right)+c . c .
\end{aligned}
$$

The total electric field, $\mathcal{E}(\mathbf{r}, t)$, is defined in terms of its frequency, $\omega$, and associated wavevector, $\mathbf{k}$, the electric field strength, $\chi$, and the field envelope, $E\left(t-\tau_{p}\right)$, centred at $\tau_{p}$, which is assumed to be Gaussian,

$$
E\left(t-\tau_{p}\right)=\exp \left(\frac{-\left(t-\tau_{p}\right)^{2}}{2 \varsigma^{2}}\right)
$$


with FWHM $=2 \sqrt{2 \ln 2} \varsigma$.

The system density matrix is initialised with the entire population in the ground electronic state,

$$
\rho(t=0)=|g\rangle\langle g|=\left(\begin{array}{ll}
1 & 0 \\
0 & 0
\end{array}\right),
$$

and is then propagated for 2.05 ps to establish systembath correlations, with $\tilde{\xi}=5000 \mathrm{~cm}^{-1}$, so that the convergence parameter is sufficiently large. The laser pulse is introduced by adding eq. 31 into the system Hamiltonian (eq. 4) during this evolution, with the pulse centred at $\tau_{p}=2$ ps. When the pulse has ended $\left(<50\right.$ fs after $\left.\tau_{p}\right)$, the ADOs are propagated for a further 2 ps, providing the states which are used in the trace distance calculations. Identical laser pulses are used for all bath conditions, with the frequency set to be resonant with the frequency of the system, $\omega_{e g}$, and $\chi=10^{3} \mathrm{Vm}^{-1}$. The two series of states for which the trace distance are calculated correspond to different FWHM of the laser pulse, where $\rho_{1}$ has a FWHM of $0 \mathrm{fs}$, corresponding to no excitation, and $\rho_{2}$ has a FWHM of $20 \mathrm{fs}$, causing significant excitation in the system.

Linear and 2D spectra are calculated using the standard response function approach, as detailed in Appendix A. with similarly correlated initial conditions, representative of experimental measurements where the systembath correlations are well established. The spectra are calculated in the impulsive limit in order to consider theory where the measurable non-Markovianity can be compared with ideal spectra, free from unwanted effects of a finite pulse shape.

\section{RESULTS AND DISCUSSION}

The trace distance, $D\left(\rho_{1}, \rho_{2}\right)$, for the range of damping strengths presented in table is shown in figure 2 (top). As in the example case of figure 1, as the excited population of $\rho_{2}$ dissipates energy to the bath, the ground state population increases, causing $\rho_{1}$ and $\rho_{2}$ to become less distinguishable and the trace distance to decrease. This description is consistent with the overall negative gradient observed for $D\left(\rho_{1}, \rho_{2}\right)$ in figure 2 .

However, the decrease in trace distance is accompanied by significant oscillations which decay over time, as shown by the flux, $\sigma$, in figure 3 . As discussed in section III positive flux corresponds to the non-Markovian return of information from the bath to the system, which is integrated in the BLP measure. Figure 2 (bottom) shows the cumulative integration of the positive flux, where the maximum value obtained is equal to $\mathcal{N}$.

From figures 2 and 3 , we observe that the $\tilde{\gamma}=50 \mathrm{~cm}^{-1}$ and $\tilde{\gamma}=300 \mathrm{~cm}^{-1}$ underdamped baths result in a slower decrease in the trace distance, with small but long-lived oscillations. This suggests that the underdamped motion enables the prolonged feedback of information from the bath to the system, with the maximum amount of measurable non-Markovianity being achieved over several
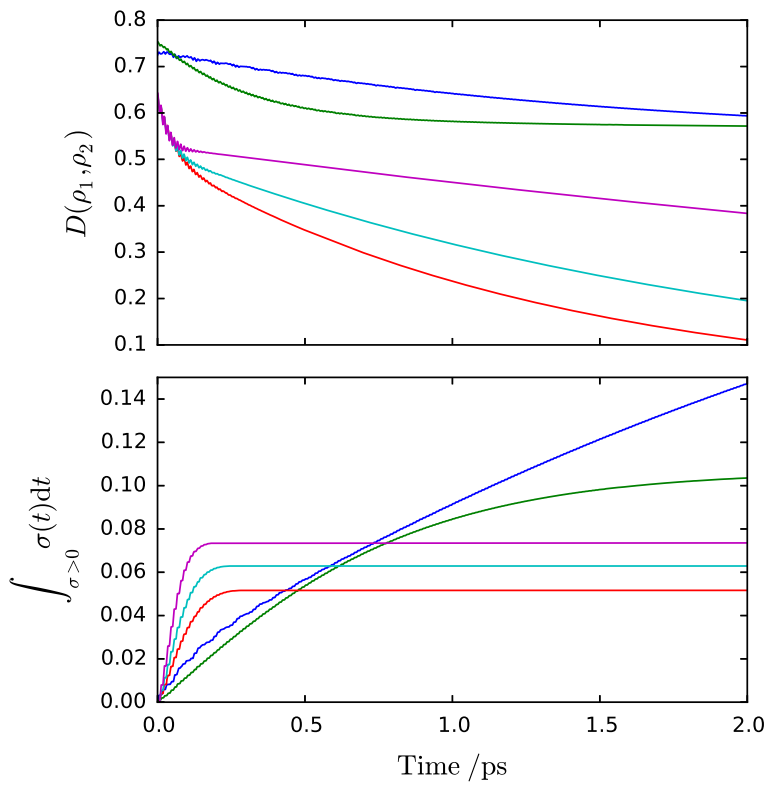

$$
\left[\begin{array}{l}
\tilde{\gamma}=50 \mathrm{~cm}^{-1} \\
-\tilde{\gamma}=300 \mathrm{~cm}^{-1} \\
-\tilde{\gamma}=1750 \mathrm{~cm}^{-1}
\end{array} \quad \begin{array}{r}
\tilde{\gamma}=2750 \mathrm{~cm}^{-1} \\
-\tilde{\gamma}=8200 \mathrm{~cm}^{-1} \\
\hline
\end{array}\right.
$$

FIG. 2. (Top) Trace distance, $D\left(\rho_{1}, \rho_{2}\right)$, and (bottom) cumulative integration of the positive flux, with maximum equal to $\mathcal{N}$, for the each of the damping strengths in table 1 .

picoseconds. There is even evidence of the wider underdamped oscillations superimposed in the positive flux, most clearly seen for $\tilde{\gamma}=50 \mathrm{~cm}^{-1}$. As the integrated flux for $\tilde{\gamma}=300 \mathrm{~cm}^{-1}$ tends towards a plateau within the time frame of our simulations, whilst $\tilde{\gamma}=50 \mathrm{~cm}^{-1}$ does not, it is clear that for an underdamped bath, increasing the damping strength decreases the maximum measurable non-Markovianity, $\mathcal{N}$.

For the remaining overdamped baths, we observe noticeably different trends. Firstly, the oscillations in the trace distance are larger and decay rapidly, within the first few hundred femtoseconds. This suggests a sudden feedback of information from the bath to the system, followed by a long period of irreversible Markovian transfer from the system to the bath. This is consistent with a sudden reorganisation of the solvent molecules upon excitation of the solute, followed by the relaxation of the excited state. Secondly, as the damping strength increases from $\tilde{\gamma}=1750 \mathrm{~cm}^{-1}$ to $\tilde{\gamma}=8200 \mathrm{~cm}^{-1}$, the measured non-Markovianity $\mathcal{N}$ also increases, achieving a plateau in the integrated flux in a shorter time. This suggests there is greater non-Markovian feedback for the inhomogeneous overdamped $\tilde{\gamma}=8200 \mathrm{~cm}^{-1}$ than the homogeneous overdamped $\tilde{\gamma}=1750 \mathrm{~cm}^{-1}$.

Therefore, figure 2 shows that by increasing the damping strength the maximum non-Markovianity is reached 

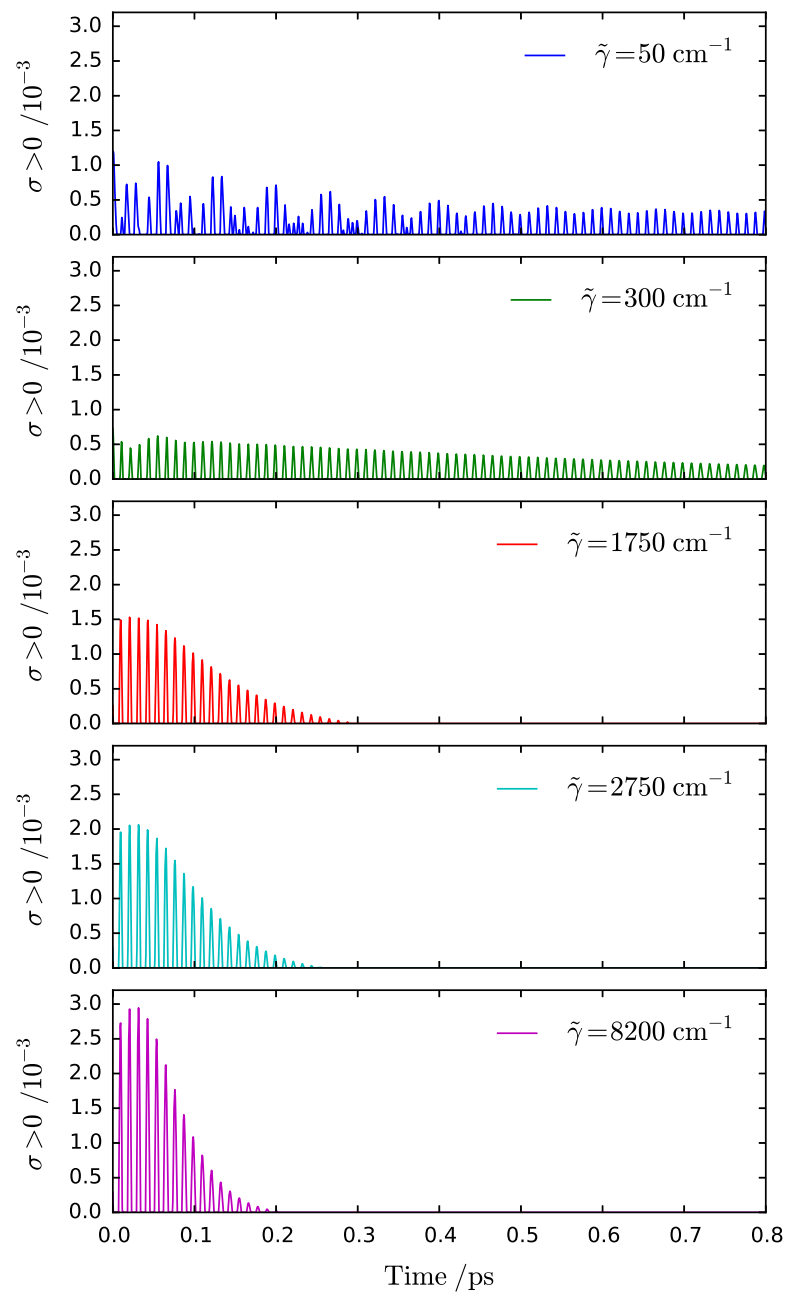

FIG. 3. Postive flux of the trace distance shown in figure 2 for each of the damping strengths in table 1

in a shorter time. This can be seen in figure 3 by the more rapid decay of positive information flux, particularly for the highly damped baths. The magnitude of maximum non-Markovianity, however, is more complicated. From figure 2 it is clear that $\mathcal{N}$ is largest in the underdamped limit due to recurrence of information being transferred back into the system from the bath over a prolonged period, as seen in figure 3. Increasing the damping then leads to a decrease in $\mathcal{N}$ for underdamped baths. But, interestingly, in the case of overdamped baths, we see that $\mathcal{N}$ increases as the damping increases from more homogeneous to more inhomogeneous environments. This suggests that broadening from an inhomogeneous environment is associated with greater nonMarkovian effects than homogeneous, but also, that potentially even greater non-Markovian effects are involved in underdamped environments, but that the total effect requires significantly longer to develop. The measured values of $\mathcal{N}$ after 2 ps are presented in table II

This change in non-Markovianity is consistent with

\begin{tabular}{c|c|c|c}
\hline$\tilde{\gamma} / \mathrm{cm}^{-1}$ & $\Delta \cdot \tau_{c}$ & $\mathcal{N}$ & FWHM $( \pm 1) / \mathrm{cm}^{-1}$ \\
\hline 50 & - & 0.147 & 4 \\
300 & - & 0.104 & 19 \\
1750 & 0.64 & 0.052 & 110 \\
2750 & 1.00 & 0.063 & 144 \\
8200 & 3.00 & 0.074 & 196 \\
\hline
\end{tabular}

TABLE II. Measured $\mathcal{N}$ and linear absorption spectrum FWHM for each damping strength in table I] where $\tilde{\eta}=$ $20 \mathrm{~cm}^{-1}$, such that $\tilde{\Delta}=91.33 \mathrm{~cm}^{-1}$ at $300 \mathrm{~K}$ for all cases. $\Delta \cdot \tau_{c}$ given for overdamped environments only.

and can be related to the observed spectral lineshape in linear and 2D spectroscopy. Linear absorption spectra for each of the damping strengths are presented in figure 4 . The $\tilde{\gamma}=50 \mathrm{~cm}^{-1}$ underdamped bath shows a sharp peak at the fundamental transition frequency, $\tilde{\omega}_{e g}$, with a vibronic peak at $\tilde{\omega}_{e g}+\tilde{\omega}_{0}$, which is significantly less intense due to the small Huang-Rhys factor of $S=0.04$. As the damping increases to $\tilde{\gamma}=300 \mathrm{~cm}^{-1}$, the vibronic peak is no longer visible and the fundamental peak broadens with a loss of intensity.

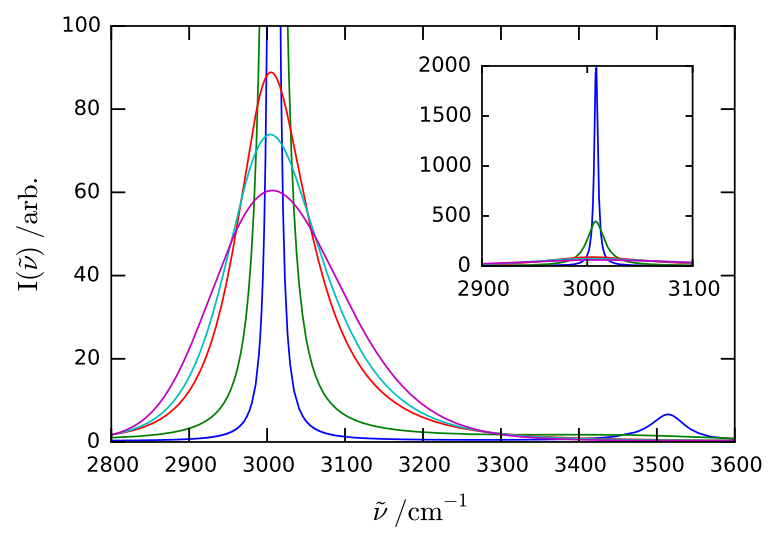

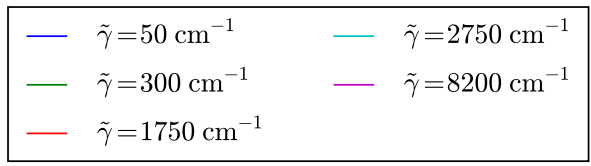

FIG. 4. Calculated linear absorption spectra for each of the damping strengths in table [

The appearance of the vibronic peak can be related to the long-lived oscillations in the trace distance and prolonged non-Markovian feedback for the underdamped baths. As the vibrational mode has been formally subsumed within the bath degrees of freedom through the canonical transformation, the observation of the vibronic peak indicates that the weak damping has enabled the spectroscopy to probe not only the system, but also the bath modes, which can only be possible via a reversible transfer of information. As the damping increases, the vibronic peak becomes much less intense, to the point of 
absence, as the feedback of information is diminished.

As the damping continues to increase to the overdamped limit, the intensity of the fundamental peak decreases further and the peak broadens from the homogeneous to the inhomogeneous limits. The FWHM of the fundamental peak for each damping strength is also listed in table II.

Recalling that the overdamped baths used in these simulations obey the high temperature limit, $k_{\mathrm{B}} T \gg \hbar \Lambda$, in the homogeneous case, $\Delta \cdot \tau_{c} \ll 1$, the absorption spectrum has a Lorentzian profile,

$$
I(\omega)=\frac{\Gamma}{\left(\omega-\omega_{e g}\right)^{2}+\Gamma^{2}},
$$

with FWHM of $2 \Gamma=2 \Delta^{2} \tau_{c}$. 1144 This corresponds to the motional narrowing limit, where the short correlation time of the bath leads to an averaging across the ensemble $!^{155}$ For $\tilde{\gamma}=1750 \mathrm{~cm}^{-1}$ with $\Delta \cdot \tau_{c}=0.64$, this predicts a FWHM of $116 \mathrm{~cm}^{-1}$, which agrees with the measured FWHM in table II] where the difference suggests that $\Delta \cdot \tau_{c}$ is too large in this case to fit the ideal homogeneous lineshape.

In the inhomogeneous case, $\Delta \cdot \tau_{c} \gg 1$, the absorption spectrum has a Gaussian profile,

$$
I(\omega)=\left(2 \pi \Delta^{2}\right)^{-\frac{1}{2}} \exp \left[-\frac{\left(\omega-\omega_{e g}\right)^{2}}{2 \Delta^{2}}\right],
$$

with FWHM of $2 \sqrt{2 \ln 2} \Delta$, directly proportional to the fluctuation amplitude ${ }^{1 / 44 \mid 45}$ The measured FWHM for the inhomogeneous $\tilde{\gamma}=8200 \mathrm{~cm}^{-1}$ with $\Delta \cdot \tau_{c}=3.00$ demonstrates a tend towards this limit of $215 \mathrm{~cm}^{-1}$.

The long correlation times associated with the inhomogeneous limit cause the bath motion to be effectively static with respect to the time scale of the system. In this way, the Gaussian profile for the linear absorption spectrum resembles the normal distribution of transition frequencies present across the ensemble due to the stochastic bath interactions. This implies a maximum amount of information about the bath is obtained, as all localised inhomogeneities contribute to the lineshape. In contrast, the short correlation times of the homogeneous limit result in seemingly identical interactions of the system and bath across the ensemble, leading to an averaging which loses this information about the bath, and produces the motional narrowing limit $\frac{15}{15}$ This agrees with the trends observed in figures 2 and 3 , where $\mathcal{N}$ is greatest and obtained fastest for the static, inhomogeneous limit. Here the maximum information of the bath is returned to the system almost instantaneously due to its slow variation. As the correlation time of the bath decreases towards the homogeneous limit, any information transferred from the system to the bath is dissipated more quickly, with less opportunity for non-Markovian feedback. This decreases the measurable non-Markovianity, as the interactions become increasingly indistinguishable and Markovian.

Early stochastic models assumed white noise, which corresponds to an entirely flat spectral density ${ }^{11114}$ This produces a correlation function which is a delta function of time, representing an instantaneous return to equilibrium for the bath, and the true Markovian limit 15 Our results demonstrate the superiority of HEOM methods in correctly accounting for coloured spectral densities and the resulting non-Markovian effects, identifying measurable non-Markovianity even as the damping approaches the homogeneous limit $37 / 46$

These non-Markovian effects are also observed using 2D spectroscopy, which separates inhomogeneous and homogeneous broadening onto the diagonal and antidiagonal of the correlation maps, respectively. Figure 5 shows the 2D spectra for population times of $T=$ $0-300$ fs for the $\tilde{\gamma}=300 \mathrm{~cm}^{-1}$ underdamped bath, identified as $\gamma<\omega_{0}$, and the three overdamped baths, labelled with their $\Delta \cdot \tau_{c}$ values. For $T=0 \mathrm{fs}$, a very intense, narrow peak is observed in the underdamped spectrum, whereas the overdamped spectra are much weaker, with significant broadening. Transitioning from the homogeneous limit of $\Delta \cdot \tau_{c}=0.64$ to the inhomogeneous limit of $\Delta \cdot \tau_{c}=3.00$, the diagonal peak width increases and the intensity decreases, reproducing the Lorentzian to Gaussian broadening observed in the linear spectra. However, the elongated diagonal width compared with the homogeneous anti-diagonal width in each of the overdamped environments confirms the presence of inhomogeneous broadening, even in the $\Delta \cdot \tau_{c}=0.64$ case. The perfectly symmetrical peak shows that no inhomogeneous broadening is observed in the underdamped environment.

On increasing the population time, spectral diffusion causes the diagonal width to decrease until it matches the anti-diagonal width. The time scale of spectral diffusion is determined by the correlation time and is a useful demonstration of the lifetime of memory effects. Whilst the population time is less than the correlation time, $T<\tau_{c}$, the inhomogeneous distribution of system-bath interactions within the ensemble contributes a series of Lorentzian peaks along the diagonal, which produce the elongated Gaussian shape when summed! ${ }^{7 / 8}$ As the population time increases, system-bath correlations are lost as the correlation function decays, decreasing this inhomogeneous broadening and leaving a more rounded peak dominated by the homogeneous lineshape ${ }^{38 / 47 / 48}$

The decay in the correlation function is directly related to the decrease in ellipticity, $E$, of the peaks in the 2D spectra, which compares the width of a Gaussian fitted to the diagonal of the peak, $\varsigma_{\mathrm{D}}$, with that of the antidiagonal, $\varsigma_{\mathrm{A}}, \underline{49} \underline{51}$

$$
E=\frac{\varsigma_{\mathrm{D}}^{2}-\varsigma_{\mathrm{A}}^{2}}{\varsigma_{\mathrm{D}}^{2}+\varsigma_{\mathrm{A}}^{2}}
$$

Figure 6 shows that the ellipticity of the three overdamped baths follows an exponential decay with population time and that the slower decay for inhomogeneous baths is accompanied by increasing $\mathcal{N}$. The homogeneous $\Delta \cdot \tau_{c}=0.64$ environment has a short correlation time such that the ellipticity has the most rapid decay and any elongation of the 2D peak due to inhomogeneous 

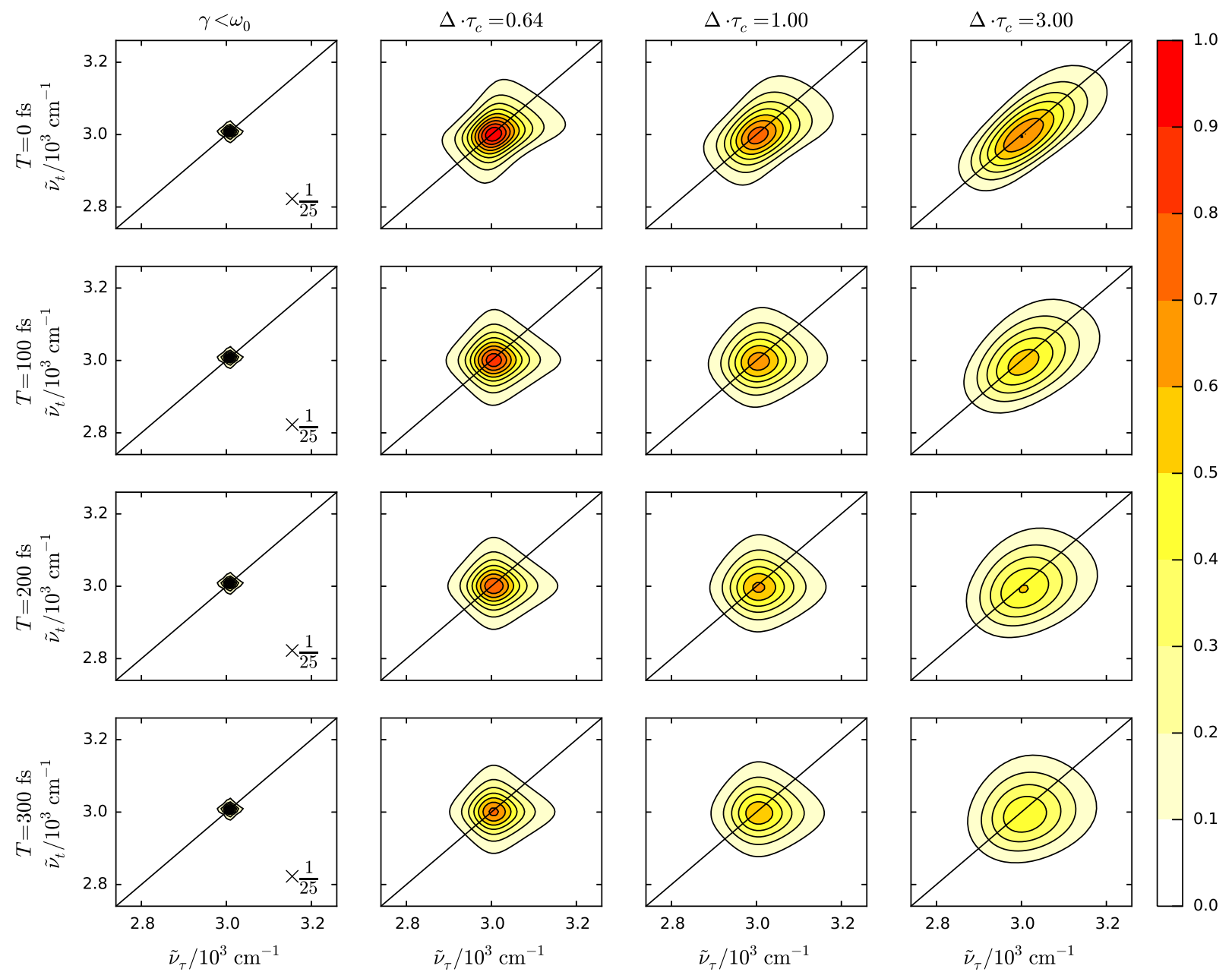

FIG. 5. Absorptive 2D spectra for population times $T=0-300 \mathrm{fs}$ for the $\tilde{\gamma}=300 \mathrm{~cm}^{-1}$ underdamped bath, labelled $\gamma<\omega_{0}$, and the three overdamped baths, identified by their $\Delta \cdot \tau_{c}$ values, as per table I normalised to the maximum of $\Delta \cdot \tau_{c}=0.64$ at $T=0$ fs.

broadening has depleted within 300 fs. The much longer correlation time of the inhomogeneous $\Delta \cdot \tau_{c}=3.00$ environment has a much slower decay in ellipticity.

Comparison with the results of the non-Markovianity measure for the overdamped environments in figures 2 and 3 suggests that the 2D lineshape can be interpreted in an identical manner to the linear spectra. As before, the short correlation time and rapid dissipation of the homogeneous bath reduce the feedback from the bath to the system, limiting any elongation due to inhomogeneous broadening and resulting in swift diffusion to a less informative 2D Lorentzian lineshape. In contrast, the much slower inhomogeneous bath, with much greater non-Markovian feedback, leads to significant elongation of the peak into a Gaussian distribution, with much slower diffusion, representing our increased knowledge of the bath degrees of freedom. The observation of greater $\mathcal{N}$ for the inhomogeneous bath in figure 2 is also related to the greater initial elongation of the peak, and thus larger $E$, at $T=0 \mathrm{fs}$ for $\Delta \cdot \tau_{c}=3.00$ compared with $\Delta \cdot \tau_{c}=0.64$ in figure 5 .

Ideally, the trends discussed above would be confirmed by performing additional simulations using parameters which further demonstrate the transitions between the damping limits. Unfortunately, the numerical integration proved insufficiently stable to traverse the critical damping region between the underdamped and overdamped limits. Similarly, identifying a suitable parameter set which could access all the limits on changing the damping strength alone presented a significant challenge. This is expected, however, as it is uncommon for a particular mode frequency with a set reorganisation energy to appear as both an underdamped and overdamped mode in nature. Underdamped modes are typically high frequency intramolecular modes with large reorganisation energies, whilst overdamped modes are lower frequency intermolecular modes with small reorganisation 

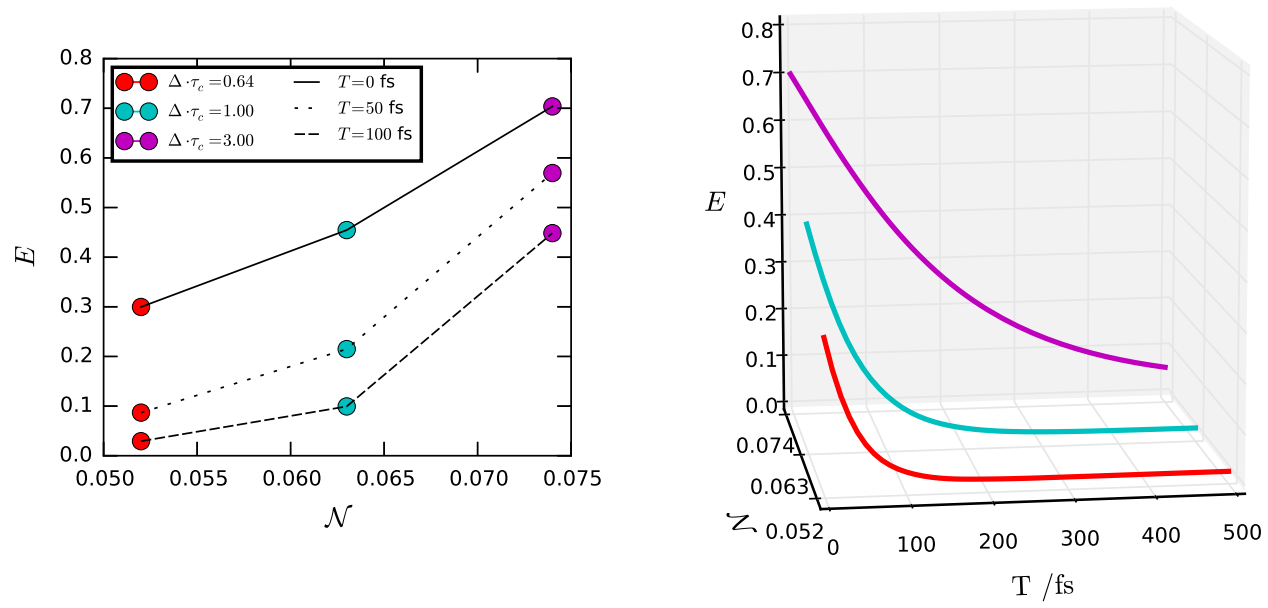

FIG. 6. Ellipticity, $E$, of the absorptive 2D spectra against the measured non-Markovianity, $\mathcal{N}$, for the three overdamped baths, identified by their $\Delta \cdot \tau_{c}$ values, for (left) $T=0,50$ and $100 \mathrm{fs}$ and (right) $T=0-400 \mathrm{fs}$, sampled at $10 \mathrm{fs}$ intervals.

energies 345253 Hence why an intermediate frequency was used in our simulations.

\section{CONCLUSION}

Here we have combined the open quantum system dynamics of a two level system coupled to a harmonic oscillator bath with metrics from quantum information theory to quantify the non-Markovianity involved in the system-bath interaction under different damping limits. We have presented separate hierarchical equations of motion methods for underdamped and overdamped environments, which adopt the latest, most efficient, termination technology, and have applied them to the relaxation dynamics of a simple system pumped by an external laser field and calculation of linear and 2D spectra.

This study shows that on decreasing the damping rate for a Brownian oscillator of fixed mode frequency and reorganization energy, the non-Markovianity measure, $\mathcal{N}$, reaches a maximum more slowly. Furthermore, the maximum value is greater as we move towards the underdamped limit because the non-monotonic slowly decaying correlation functions provide a high flux of information feedback from the bath to the system. This is not the case for the monotonically decaying correlation functions associated with overdamped baths, where information is rapidly lost from the system with little recurrence. More concisely stated, underdamped environments have the potential for greater non-Markovian effects, but in general it takes a much longer time for the maximal amount of information to return to the system from the bath.

These results have then been compared with the changes in lineshape of the linear and 2D spectra of these systems, analysing the spectral features in terms of the information flow between the system and its bath. We quantitatively link non-Markovian effects, associated with $\mathcal{N}$, to inhomogeneous broadening and ellipticity such that this broadening can be directly interpreted as feedback of information from the bath to the system. This demonstrates that environmental signatures emerge in spectral lineshape as a result of information flow from the bath back into the system.

\section{ACKNOWLEDGMENTS}

The research presented in this paper was carried out on the High Performance Computing Cluster supported by the Research and Specialist Computing Support service at the University of East Anglia. D. G. and B. S. H. thank the Faculty of Science, University of East Anglia for studentship funding.

\section{Appendix A: Linear and 2D Spectroscopy Calculations}

The linear and non-linear spectra are calculated using the standard response function method in the impulsive limit!138 The linear absorption spectrum, $\sigma_{A}(\omega)$, is obtained by Fourier transformation of the first order molecular response function, $R^{(1)}(t)$

$$
\sigma_{A}(\omega)=\int_{0}^{\infty} \mathrm{d} t e^{i \omega t} i R^{(1)}(t)
$$




$$
R^{(1)}(t)=\frac{i}{\hbar} \operatorname{Tr}\left(\hat{\mu} \hat{G}\left(t, t_{0}\right) \hat{\mu}^{\times} \rho(-\infty)\right),
$$

where $\rho(-\infty)$ is the equilibrium density matrix and $\hat{G}\left(t_{1}, t_{0}\right)=e^{\mathcal{L}\left(t_{1}-t_{0}\right)}$ is the hierarchical propagator (eq. 17 and 29) 15116134 The equilibrium density matrix is obtained by propagating the HEOM for $2 \mathrm{ps}$ in order to establish initial correlations in the ADOs due to systembath coupling .54 The dipole moment operator is applied to all ADOs contained within $\rho(-\infty) ! \underline{34}$

Similarly, distinguishing the raising $\left(\hat{\mu}_{\rightarrow}\right)$ and lowering $\left(\hat{\mu}_{\leftarrow}\right)$ contributions to the dipole moment operator, $\hat{\mu}_{\rightleftarrows}=$ $\sigma_{\mp}$, the rephasing, $R_{\mathrm{R}}^{(3)}$, and non-rephasing, $R_{\mathrm{NR}}^{(3)}$, third order molecular response functions are given by, 54

$$
\begin{aligned}
& R_{\mathrm{R}}^{(3)}(\tau, T, t)=-\operatorname{Tr}\left(\hat{\mu} \hat{G}(t+T+\tau, T+\tau) \frac{i}{\hbar} \hat{\mu}_{\rightarrow}^{\times} \hat{G}(T+\tau, \tau) \frac{i}{\hbar} \hat{\mu}_{\rightarrow}^{\times} \hat{G}\left(\tau, t_{0}\right) \frac{i}{\hbar} \hat{\mu}_{\leftarrow}^{\times} \rho(-\infty)\right), \\
& R_{\mathrm{NR}}^{(3)}(\tau, T, t)=-\operatorname{Tr}\left(\hat{\mu} \hat{G}(t+T+\tau, T+\tau) \frac{i}{\hbar} \hat{\mu}_{\rightarrow}^{\times} \hat{G}(T+\tau, \tau) \frac{i}{\hbar} \hat{\mu}_{\leftarrow}^{\times} \hat{G}\left(\tau, t_{0}\right) \frac{i}{\hbar} \hat{\mu}_{\rightarrow}^{\times} \rho(-\infty)\right) .
\end{aligned}
$$

2D spectra are then obtained as the double Fourier transformation of the third order response function for each component,

$$
S_{\mathrm{R}}\left(\omega_{\tau}, T, \omega_{t}\right)=\int_{0}^{\infty} \mathrm{d} t \int_{0}^{\infty} \mathrm{d} \tau e^{-i \omega_{\tau} \tau} e^{i \omega_{t} t} i R_{\mathrm{R}}^{(3)}(\tau, T, t),
$$

$$
S_{\mathrm{NR}}\left(\omega_{\tau}, T, \omega_{t}\right)=\int_{0}^{\infty} \mathrm{d} t \int_{0}^{\infty} \mathrm{d} \tau e^{i \omega_{\tau} \tau} e^{i \omega_{t} t} i R_{\mathrm{NR}}^{(3)}(\tau, T, t),
$$

which produce the absorptive spectrum when summed, $\underline{3437}$

$$
S_{\mathrm{A}}=\boldsymbol{\operatorname { R e }}\left(S_{\mathrm{R}}+S_{\mathrm{NR}}\right)
$$

${ }^{1}$ S. Mukamel, Principles of Nonlinear Optical Spectroscopy (Oxford University Press, New York, 1995).

${ }^{2}$ D. M. Jonas, Annu. Rev. Phys. Chem. 54, 425 (2003)

${ }^{3}$ Y. Tanimura and A. Ishizaki, Acc. Chem. Res. 42, 1270 (2009)

${ }^{4}$ J. C. Dean and G. D. Scholes, Acc. Chem. Res. 50, 2746 (2017)

${ }^{5}$ T. l. C. Jansen, S. Saito, J. Jeon, and M. Cho, J. Chem. Phys. 150, 100901 (2019).

${ }^{6}$ S. Mukamel, Y. Tanimura, and P. Hamm, Acc. Chem. Res. 42, 1207 (2009)

${ }^{7}$ M. Khalil, N. Demirdöven, and A. Tokmakoff, J. Phys. Chem. A 107, 5258 (2003)

${ }^{\gamma}$ A. Gelzinis, R. Augulis, V. Butkus, B. Robert, and L. Valkunas, Biochim. Biophys. Acta, Bioenerg. 1860, 271 (2019)

${ }^{9}$ H.-P. Breuer and F. Petruccione, The Theory of Open Quantum Systems (Oxford University Press, 2007).

${ }^{10}$ I. de Vega and D. Alonso, Rev. Mod. Phys. 89, 015001 (2017)

${ }^{11}$ Y. Tanimura and R. Kubo, J. Phys. Soc. Jpn. 58, 101 (1989).

${ }^{12}$ U. Weiss, Quantum Dissipative Systems, 4th ed. (World Scientific, 2012).

${ }^{13}$ P. Kjellberg, B. Brüggemann, and T. Pullerits, Phys. Rev. B 74, 024303 (2006)

${ }^{14}$ Y. Tanimura, Phys. Rev. A 41, 6676 (1990)

${ }^{15}$ Y. Tanimura, J. Phys. Soc. Jpn. 75, 082001 (2006)

${ }^{16}$ Y. Tanimura, J. Chem. Phys. 142, 144110 (2015)

${ }^{17}$ M. Tanaka and Y. Tanimura, J. Chem. Phys. 132, 214502 (2010)

${ }^{18}$ C. Kreisbeck, T. Kramer, and A. Aspuru-Guzik, J. Chem. Theory Comput. 10, 4045 (2014)
${ }^{19}$ M. Schröter, T. Pullerits, and O. Kühn, Ann. Phys. (Berlin, Ger.) 527, 536 (2015)

${ }^{20}$ T. Kramer, M. Noack, A. Reinefeld, M. Rodríguez, and Y. Zelinskyy, J. Comput. Chem. 39, 1779 (2018)

${ }^{21}$ H.-P. Breuer, E.-M. Laine, J. Piilo, and B. Vacchini, Rev. Mod. Phys. 88, 021002 (2016)

${ }^{22}$ A. Rivas, S. F. Huelga, and M. B. Plenio, Rep. Prog. Phys. 77, 094001 (2014)

${ }^{23}$ A. G. Dijkstra and Y. Tanimura, Phil. Trans. R. Soc. A 370, 3658 (2012)

${ }^{24}$ H.-P. Breuer, E.-M. Laine, and J. Piilo, Phys. Rev. Lett. 103, 210401 (2009)

${ }^{20}$ E.-M. Laine, J. Piilo, and H.-P. Breuer, EPL 92, 60010 (2010)

${ }^{26}$ E.-M. Laine, J. Piilo, and H.-P. Breuer, Phys. Rev. A 81, 062115 (2010)

${ }^{2 /}$ S. Mukamel, Annu. Rev. Phys. Chem. 41, 647 (1990)

${ }^{28}$ M. A. Nielsen and I. L. Chuang, Cambridge University Press (Cambridge University Press, Cambridge, 2010).

${ }^{29}$ A. Wehrl, Rev. Mod. Phys. 50, 221 (1978)

${ }^{30}$ P. M. Poggi, F. C. Lombardo, and D. A. Wisniacki, EPL 118, $20005(2017)$

${ }^{31}$ X.-M. Lu, X. Wang, and C. P. Sun, Phys. Rev. A 82, 042103 (2010)

${ }^{32}$ H.-S. Zeng, N. Tang, Y.-P. Zheng, and G.-Y. Wang, Phys. Rev. A 84, 032118 (2011)

${ }^{35}$ B. Witt, L. Rudnicki, Y. Tanimura, and F. Mintert, New J. Phys. 19, 013007 (2017)

${ }^{34}$ Y. Tanimura, J. Chem. Phys. 137, 22A550 (2012)

${ }^{35}$ M. Tanaka and Y. Tanimura, J. Phys. Soc. Jpn. 78, 073802 (2009)

${ }^{36}$ A. G. Dijkstra and V. I. Prokhorenko, J. Chem. Phys. 147, $064102(2017)$

'A. Ishizaki and Y. Tanimura, Chem. Phys. 347, 185 (2008).

${ }^{38} \mathrm{P}$. Hamm and M. Zanni, Concepts and Methods of $2 D$ Infrared Spectroscopy (Cambridge University Press, Cambridge, 2011).

${ }^{39}$ A. G. Dijkstra and Y. Tanimura, J. Chem. Phys. 142, 212423 (2015)

${ }^{40}$ A. G. Dijkstra and Y. Tanimura, J. Phys. Soc. Jpn. 81, 063301 (2012)

${ }^{41}$ D. Green, F. V. A. Camargo, I. A. Heisler, A. G. Dijkstra, and G. A. Jones, J. Phys. Chem. A 122, 6206 (2018)

${ }^{42}$ M. F. Gelin, D. Egorova, and W. Domcke, J. Chem. Phys. 123, $164112(2005)$

${ }^{43}$ X. Leng, S. Yue, Y.-X. Weng, K. Song, and Q. Shi, Chem. Phys. Lett. 667, 79 (2017)

${ }^{44}$ J. Sue, Y. J. Yan, and S. Mukamel, J. Chem. Phys. 85, 462 (1986)

${ }^{45}$ O. Kühn, V. Rupasov, and S. Mukamel, J. Chem. Phys. 104, 
$5821(1996)$

${ }^{40}$ A. Ishizaki and Y. Tanimura, J. Phys. Soc. Jpn. 74, 3131 (2005)

${ }^{47}$ A. Tokmakoff, J. Phys. Chem. A 104, 4247 (2000)

${ }^{48}$ F. Šanda, V. Perlík, C. N. Lincoln, and J. Hauer, J. Phys. Chem.

A 119, 10893 (2015)

${ }^{49}$ K. Lazonder, M. S. Pshenichnikov, and D. A. Wiersma, Opt. Lett. 31, 3354 (2006)

${ }^{50}$ S. T. Roberts, J. J. Loparo, and A. Tokmakoff, J. Chem. Phys. 125, 084502 (2006)
${ }^{51}$ P. J. Nowakowski, M. F. Khyasudeen, and H.-S. Tan, Chem. Phys. 515, 214 (2018)

${ }^{52}$ S. M. Gallagher Faeder and D. M. Jonas, J. Phys. Chem. A 103, 10489 (1999)

${ }^{53}$ J. Iles-Smith, A. G. Dijkstra, N. Lambert, and A. Nazir, J. Chem. Phys. 144, 044110 (2016).

${ }^{54}$ A. Ishizaki and Y. Tanimura, J. Phys. Chem. A 111, 9269 (2007) 